\title{
A ROLE FOR DOUBLY STOCHASTIC MATRICES IN GRAPH THEORY
}

\author{
D. J. HARTFIEL AND J. W. SPELLMANN
}

\begin{abstract}
This paper represents a strongly connected digraph as a doubly stochastic matrix. It then uses this doubly stochastic representation to prove several theorems concerning the critical arcs of strongly connected graphs.
\end{abstract}

Definitions and notation. The definitions concerning matrix theory for the most part may be found in [3]. All others will be given below.

An $n \times n$ nonnegative matrix $A=\left(a_{i j}\right)$, so that $\sum_{R} a_{i R}=\sum_{R} a_{R i}=\lambda$ for each $i \in\{1,2, \cdots, n\}$, is called a $\lambda$-doubly stochastic matrix. If $\lambda=1$, the matrix is simply called doubly stochastic. A pair of matrices $A=\left(a_{i j}\right)$ and $B=\left(b_{i j}\right)$ such that $a_{i j}=0$ if and only if $b_{i j}=0$ are said to have the same 0 -pattern. If there is a doubly stochastic matrix $B$ which has the same 0 -pattern as $A$, then $A$ is said to have doubly stochastic pattern. We say that $A$ has a loop if $A$ has distinct positive entries

$$
a_{i_{1} j_{1}}, a_{i_{2} j_{2}}, a_{i_{2} j_{2}}, a_{i_{2} j_{3}}, \cdots, a_{i_{r} j_{r}}, a_{i_{r} j_{r+1}}, a_{i_{r+1} j_{r+1}}=a_{i_{1} j_{1}} .
$$

Finally, let $E_{i j}$ denote the $n \times n(0,1)$-matrix with a one only in the $i j$ th position.

All definitions concerning graph theory can be found in [1] and [5].

Results. We first establish a relation between doubly stochastic matrices and digraphs. For this we include the following two theorems.

THEOREM 1. A digraph $G$ is strongly connected if and only if its associated matrix $A$ is irreducible $[\mathbf{1 0}$, p. 20].

THEOREM 2. If $A$ is an irreducible matrix, then there is a diagonal matrix $D$ with positive main diagonal so that $D A D^{-1}$ has its ith row sum equal to its ith column sum for each $i \in\{1,2, \cdots, n\}[6$, p. 3].

Now a positive main diagonal may be added to the matrix $D A D^{-1}$ to yield a $\lambda$-doubly stochastic matrix where $\lambda>1$. Hence the relation between doubly stochastic matrices and digraphs may be stated as:

Received by the editors May 18, 1971.

AMS (MOS) subject classifications (1970). Primary 05C20, 05B20; Secondary 05C35, $15 \mathrm{~A} 51$.

Key words and phrases. Digraph, doubly stochastic matrices, critical arcs.

(c) American Mathematical Society 1973 
THEOREM 3. A digraph is strongly connected if and only if there is a doubly stochastic matrix $M$ with positive main diagonal so that

(a) $M$ is irreducible, and

(b) $M=\left(m_{i j}\right)$ is such that if $i \neq j$ then $m_{i j}>0$ if and only if there is an arc from $x_{i}$ to $x_{j}$.

Any such matrix $M$ is called a doubly stochastic model of $G$. We now give three lemmas concerning doubly stochastic matrices.

Lemma 1. Suppose $A$ is nonnegative with a positive main diagonal. Then the following two statements are equivalent.

(a) A has doubly stochastic pattern.

(b) There is a permutation matrix $P$ so that $P A P^{T}$ is a direct sum of irrreducible matrices, i.e. $A$ is completely reducible.

Proof. We first show that (a) implies (b). For this we may assume that $A$ is doubly stochastic. If $A$ is irreducible we are through. If $A$ is reducible, then there is a permutation matrix $P$ so that $P A P^{T}=\left(\begin{array}{ll}X & A \\ Z & Y\end{array}\right)$ where $X$ and $Y$ are square. Suppose $X$ is $k \times k$. Then $\sum_{i, j} x_{i j}=k$ by considering the first $k$ row sums of $P A P^{T}$. Considering the first $k$ column sums of $P A P^{T}$ we have $\sum_{i, j} x_{i j}+\sum_{i, j} z_{i j}=k$. Hence $\sum_{i, j} z_{i j}=0$ and $P A T^{T}=\left(\begin{array}{cc}X & 0 \\ 0 & Y\end{array}\right)$. Since $X$ and $Y$ are now doubly stochastic the theorem follows by induction.

That (b) implies (a) is an application of Theorem 2.

LEMMA 2. If $A$ is an irreducible doubly stochastic matrix with a positive main diagonal and $i \neq j$, then the following two statements are equivalent.

(a) $A-a_{i j} E_{i j}$ has doubly stochastic pattern.

(b) $A-a_{i j} E_{i j}$ is irreducible.

Proof. We first show that (a) implies (b). If $A-a_{i j} E_{i j}$ has doubly stochastic pattern then by Lemma 1 it is irreducible or completely reducible. If $A-a_{i j} E_{i j}$ is completely reducible, then $A$ is reducible. Hence $A-a_{i j} E_{i j}$ must be irreducible.

That (b) implies (a) is a consequence of Theorem 2 .

LEMMA 3. If $A$ is a doubly stochastic matrix with positive main diagonal then there is a doubly stochastic matrix $B$ with the same 0-pattern as $A$ so that each main diagonal entry of $B$ is larger than each nondiagonal entry of $B$.

Proof. Suppose $\lambda$ is some number so that $0<\lambda<1$. Then $\lambda A+(1-\lambda) I$ is doubly stochastic with the same 0 -pattern as $A$. Hence, for $\lambda$ sufficiently small, Lemma 3 follows. 
We are now in a position to show how doubly stochastic matrices may be used in the analysis of critical arcs in strongly connected digraphs.

THEOREM 4. The arc $x_{i j}$ from vertex $v_{i}$ to vertex $v_{j}$ of a strongly connected digraph $G$ is critical if and only if there is not a doubly stochastic model $M$ of $G$ with $m_{i j}$ smaller than all other nonzero entries in $M$.

Proof. Suppose $x_{i j}$ is not critical. Then $G-x_{i j}$ is still strongly connected. Hence there is a doubly stochastic model $N=\left(n_{i j}\right)$ of $G-x_{i j} . x_{i j}$ is on an elementary circuit in $G$, say $x_{i j}, x_{j j_{1}}, x_{j_{1} j_{2}}, \cdots, x_{j_{R}}$. Suppose $\varepsilon>0$. Add $\varepsilon$ to $n_{i j}, n_{j j_{1}}, n_{j_{1} j_{2}}, \cdots, n_{j_{R^{i}}}$ in $N$ and subtract $\varepsilon$ from $n_{i i}, n_{j j}$, $n_{j_{1} j_{1}}, \cdots, n_{j_{R^{j}}}$ in $N$ yielding the matrix $N^{\prime}=\left(n_{i j}^{\prime}\right)$. For $\varepsilon$ sufficiently small, $N^{\prime}$ is a doubly stochastic model of $G$ and $n_{i j}$ is smaller than all other nonzero entries in $M$.

Suppose $x_{i j}$ is critical. Further suppose $G$ has a doubly stochastic model $M$ so that $m_{i j}$ is smaller than all other nonzero entries in $M$. Subtract $m_{i j}$ from $m_{i j}, m_{j_{1}}, \cdots, m_{j_{R^{i}}}$ in $M$ and add $m_{i j}$ to $m_{i i}, m_{j j}, m_{j_{1} j_{1}}, \cdots$, $m_{j_{R} j_{n}}$ in $M$ yielding the matrix $M^{\prime} . M^{\prime}$ is a doubly stochastic model of $G-x_{i j}$. Lemma 2 then implies that $M^{\prime}$ is irreducible. Hence $G-x_{i j}$ is strongly connected. This contradicts the hypothesis that $x_{i j}$ is critical.

THEOREM 5. If $G$ is a strongly connected digraph, then given any elementary circuit $C$ of $G$ there is a nonempty set of arcs from $C$ whose removal from $G$ leaves

(a) a strongly connected digraph, or

(b) a disjoint union of strongly connected digraphs.

Proof. Suppose the elementary circuit $C$ contains the following arcs: $x_{i_{1} i_{2}}, x_{i_{2} i_{3}}, \cdots, x_{i_{R^{i_{1}}}}$. Suppose $M$ is a doubly stochastic model of $G$. By Lemma 3 we may assume every entry $m_{R R}$ of $M$ is larger than every entry $m_{i j}(i \neq j)$ of $M$. Now consider $m_{i_{1} i_{2}}, m_{i_{2} i_{3}}, \cdots, m_{i_{R^{i} i_{1}}}$ in $M$. Let $m=$ $\min \left[m_{i_{1} i_{2}}, \cdots, m_{i_{R} i_{1}}\right]$. Now subtract $m$ from each of $m_{i_{1} i_{2}}, m_{i_{2} i_{3}}, \cdots, m_{i_{R} i_{1}}$ in $M$ and add $m$ to $m_{i_{1} i_{1}}, m_{i_{2} i_{2}}, \cdots, m_{i_{R} i_{R}}$ in $M$. This results in a doubly stochastic matrix $M^{\prime}$. The result now follows from Theorem 3 and Lemma 1 .

Note that the theorem does not imply that any of the arcs of $C$ may be arbitrarily chosen as can be seen by considering the example below:

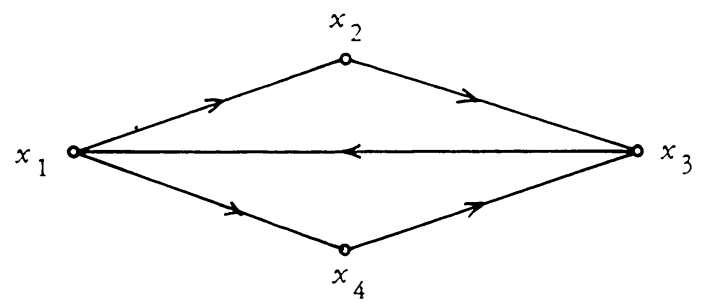


Let $C: x_{14}, x_{43}, x_{31}$. The arcs $x_{14}$ and $x_{43}$ may be removed to yield two disjoint strongly connected digraphs, i.e.

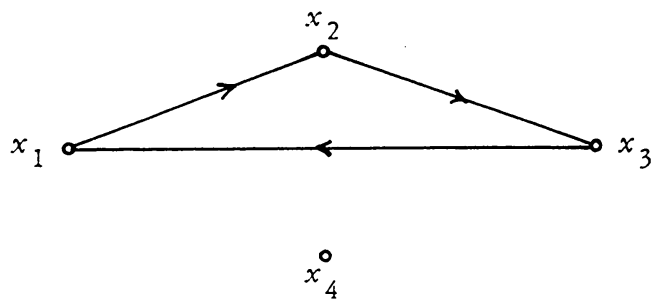

however, $x_{31}$ may not be removed. An implication of this theorem is, of course, that a minimally strongly connected digraph is the connection through an elementary circuit of minimally strongly connected digraphs with fewer vertices.

The next theorem of this section is also in [4, p. 18]; however, we feel that our proof is the more elementary.

THEOREM 6. Suppose $G$ is a minimally connected digraph with symmetric $\operatorname{arcs} x_{i j}$ and $x_{j i}$. If $x_{i j}$ and $x_{j i}$ are removed from $G$ yielding $G^{\prime}$, then $G^{\prime}$ is a disjoint union of two strongly connected digraphs.

Proof. Let $M$ be a doubly stochastic model of $G$. Suppose $\varepsilon$ is a positive number with $0<\varepsilon<\min \left[m_{i j}, m_{j i}\right]$. Subtract $\varepsilon$ from $m_{i j}$ and $m_{j i}$ in $M$ and add $\varepsilon$ to $m_{i i}$ and $m_{j j}$ in $M$. This yields a doubly stochastic matrix $M^{\prime}$ which is a model for $G$. Further for sufficiently large $\varepsilon$ we see that $m_{i j}^{\prime}$ or $m_{j i}^{\prime}$ is a smallest positive entry in $M^{\prime}$. Without loss of generality suppose $m_{i j}^{\prime} \leqq m_{j i}^{\prime}$. Since $x_{i j}$ is critical, $M^{\prime}-m_{i j}^{\prime} E_{i j}$ is reducible. Hence there is a permutation matrix $P$ so that $P\left[M-m_{i j}^{\prime} E_{i j}\right] P^{T}=\left(\begin{array}{cc}X & \stackrel{0}{Z}\end{array}\right)$ where $X$ and $Y$ are square. Suppose $X$ is $k \times k$. Then $\sum_{i j} x_{i j}=k-m_{i j}^{\prime}$. Further $\sum_{i j} x_{i j}+$ $\sum_{i j} z_{i j}=k$. Hence $\sum_{i j} z_{i j}=m_{i j}^{\prime}$. Since $m_{j i}^{\prime}$ is in $Z$ we see that $Z$ has only one positive entry. Hence the removal of $x_{i j}$ and $x_{j i}$ from $G$ results in two disjoint digraphs. It is easily seen that these two digraphs are strongly connected and hence the theorem is established.

We include the next two theorems to show some slightly different techniques of argument in the use of doubly stochastic matrices.

THEOREM 7. Suppose $G$ is a strongly connected digraph with $C_{1}$ and $C_{2}$ elementary circuits of $G$. Suppose $C_{1}$ and $C_{2}$ have one and only one arc $x$ in common. Then there is a nonempty set of arcs $S$ of $C_{1}$ so that $x \notin S$ and

(a) $G-S$ is a strongly connected digraph, or

(b) $G-S$ is a disjoint union of strongly connected digraphs. 
Proof. Suppose $M$ is a doubly stochastic model of $G$ so that $M$ is $n \times n$ and $m_{i i}>m_{p q}$ for all $i, p, q \in\{1,2, \cdots, n\}$ with $p \neq q$. Suppose $C_{1}: x_{i_{1} i_{2}}, x_{i_{2} i_{3}}, x_{i_{R} i_{1}} ; C_{2}: x_{j_{1} j_{2}}, x_{j_{2} j_{3}}, \cdots, x_{j_{S} j_{1}}$. Let

$$
m=\max \left\{m_{i_{1} i_{2}}, m_{i_{2} i_{3}}, \cdots, m_{i_{R} i_{1}}\right\} \text {. }
$$

Now add $m$ to $m_{j_{1} j_{2}}, m_{j_{2} j_{3}}, \cdots, m_{j_{s^{j}}}$ in $M$ and subtract $m$ from $m_{j_{1} j_{1}}$, $m_{j_{2} j_{2}}, \cdots, m_{j_{S} j_{S}}$ obtaining a doubly stochastic model $M^{\prime}$ of $G . M^{\prime}$ has the property that $\max \left\{m_{i_{1} i_{2}}^{\prime}, m_{i_{2} i_{3}}^{\prime}, \cdots, m_{i_{R} i_{1}}^{\prime}\right\}=m_{i j}^{\prime}$ where $x_{i j}=x$. Further, $m_{i j}^{\prime}$ is larger than every other number in $\left\{m_{i_{1} i_{2}}^{\prime}, m_{i_{2} i_{3}}^{\prime}, \cdots, m_{i_{R_{1}}}^{\prime}\right\}$. The result now follows by an argument similar to that of Theorem 5 .

THEOREM 8. If $G$ is a strongly connected digraph and $C$ a closed semipath in $G$ then there is a nonempty set of arcs $S$ from $C$ so that

(a) $G-S$ is a strongly connected digraph, or

(b) $G-S$ is a disjoint union of strongly connected digraphs.

Proof. Let $M$ be a doubly stochastic model of $G$ so that every main diagonal entry is larger than every off diagonal entry. Let $C: v_{i_{1}}, x_{i_{1} j_{1}}, v_{i_{2}}$, $x_{i_{2} j_{2}}, \cdots, v_{i_{S}}, x_{i_{S} j_{S}}, v_{i_{1}}$ where $v_{i_{R}}$ each is a vertex of $G$ and $x_{i_{R^{j}}}$ an arc between $v_{i_{R}}$ and $v_{i_{R+1}}(k+1 \bmod S)$. Consider the following entries in $M$ : $\left\{m_{i_{R} j_{R}}\right.$ where $\left.R \in\{1,2, \cdots, S\}\right\} \cup\left\{m_{i_{R^{i}}{ }^{2}}\right.$ if $x_{i_{R-1} j_{R-1}}$ is an arc into $v_{R}$ and $x_{i_{R} j_{R}}$ an arc out of $\left.v_{R}\right\}=N$. Now the entries of $N$ in $M$ form a loop of $M: m_{t_{1} t_{2}}, m_{t_{3} t_{2}}, M_{t_{3} t_{4}}, \cdots, m_{t_{r} t_{r+1}}, m_{t_{r_{2}} t_{r+1}}, m_{t_{r_{+}} t_{r_{r+3}}}=m_{t_{1} t_{2}}$. Again, without loss of generality we may assume $\min _{m_{i} \in \mathrm{E} . \mathrm{V}}\left\{m_{i j}\right\}=m_{t_{1} t_{2}}$. Subtract $m_{t_{1} t_{2}}$ from each of $m_{t_{1} t_{2}}, m_{t_{3} t_{4}}, \cdots, m_{t_{r} t_{r+1}}$ in $M$ and add $m_{t_{1} t_{2}}$ to each of $m_{t_{3} t_{2}}, \cdots$, $m_{t_{r+2} t_{r+1}}$ in $M$ yielding the matrix $M^{\prime} . M^{\prime}$ is now a doubly stochastic model of $G-S$ and the result follows immediately.

The above two theorems may well shed some additional light on the structure of minimally connected digraphs.

In conclusion we list [2], [7], and [9] as papers relevant to our topic. Brualdi, in [2], gives an extensive discussion of the kinds of row and column sums which can be obtained when the 0-pattern of a matrix is specified. Sinkhorn and Knopp in [9] give an interesting substructure of fully indecomposable matrices through the use of doubly stochastic matrices.

\section{BIBLIOGRAPHY}

1. Claude Berge, Théorie des graphes et ses applications, Dunod, Paris, 1958; English transl., Wiley, New York, 1962. MR 21 \#1608; MR 24 \#A2381.

2. R. A. Brualdi, Convex sets of non-negative matrices, Canad. J. Math. 20 (1968), 144-157. MR 36 \#2636.

3. F. R. Gantmaher, The theory of matrices, GITTL, Moscow, 1953; English transl., Chelsea, New York, 1959. MR 16, 438; MR 21 \#6372c.

4. Dennis P. Geller, Minimally strong digraphs, Proc. Edinburgh Math. Soc. (2) 17 (1970), 15-22. MR 42 \#1718. 
5. Frank Harary, Graph theory, Addison-Wesley, Reading, Mass., 1969. MR 41 \#1566.

6. D. J. Hartfiel, Concerning diagonal similarity of irreducible matrices, Proc. Amer. Math. Soc. 30 (1971), 419-425.

7. W. B. Jurkat and H. J. Ryser, Term rank and permanents of nonnegative matrices, J. Algebra 5 (1967), 342-357. MR 35 \#6575.

8. H. E. Robbins, $A$ theorem on graphs, with an application to a problem of traffic control, Amer. Math. Monthly 46 (1939), 281-283.

9. Richard Sinkhorn and Paul Knopp, Problems involving diagonal products in nonnegative matrices, Trans. Amer. Math. Soc. 136 (1969), 67-75. MR 38 \#2151.

10. Richard S. Varga, Matrix iterative analysis, Prentice-Hall, Englewood Cliffs, N.J., 1962. MR 28 \#1725.

Department of Mathematics, Texas a\&M University, College Station, TEXAS 77843

Current address (J. W. Spellmann): Department of Mathematics, Pan American University, Edinburg, Texas 78539 